\title{
Análise espacial da taxa de pobreza e da população rural da região sul do país
}

\section{RESUMO}

O presente artigo verifica a existe relação espacial entre a taxa de pobreza e o percentual da população rural nos municípios da região Sul do Brasil. O trabalho utiliza a Análise Exploratória dos Dados Espaciais (AEDE) para identificar os coeficientes bivariados I de Moran. Os dados são fornecidos pelo Instituto Brasileiro de Geografia e Estatística (IBGE). Pode-se verificar através da (AEDE), que entre os anos de 2000 e de 2010, houve aumento pouco expressivo da autocorrelação positiva entre o percentual da população rural e taxa de pobreza para os municípios da região Sul. Os resultados indicaram que os municípios com maiores percentuais de população rural e taxa de pobreza acima da média estão concentrados nas regiões Central do Paraná e do Rio Grande do Sul, já a concentração de municípios com os menores percentuais de pobres e de população rural estão localizados nas regiões do Norte do Paraná e Metropolitana de Porto Alegre nos dois anos analisados. Verificou-se que ocorreu um aumento pouco expressivo da autocorrelação espacial positiva entre o percentual da população rural e taxa de pobreza para os municípios do Sul do Brasil.

Palavra-chave: Pobreza; AEDE; Rural

\begin{abstract}
This article verifies the spatial relationship between the poverty rate and the percentage of the rural population in the municipalities of the southern region of Brazil. The work uses the Spatial Data Exploratory Analysis (AEDE) to identify the bivariate coefficients I of Moran. The data are provided by the Brazilian Institute of Geography and Statistics (IBGE). It can be verified from AEDE that between 2000 and 2010 there was a small expressive increase in the positive autocorrelation between the percentage of the rural population and the poverty rate for the municipalities of the South region. The highest percentages of the rural population and above average poverty rates are concentrated in the Central regions of Paraná and Rio Grande do Sul, and the concentration of municipalities with the lowest percentages of poor and rural population are located in the regions of Northern Paraná and Metropolitan of Porto Alegre in the two years analyzed. It was verified that there was a little expressive increase of the positive spatial autocorrelation between the percentage of the rural population and poverty rate for the municipalities of the South of Brazil.
\end{abstract}

Keyword: Poverty; AEDE; RURAL 


\section{INTRODUÇÃO}

A pobreza é um problema que tem intrigado formuladores de política econômica e estudiosos das áreas sociais. No ano de 2000 entre um dos oito objetivos do milênio levantados pela Organização das Nações Unidas estava a erradicação da pobreza e da fome. De acordo com o relatório do Banco Mundial de 2008 houve redução da taxa de pobreza em muitos dos países em desenvolvimento, mas o fenômeno continua sendo predominantemente rural, com mais de $80 \%$ dos pobres vivendo nestas áreas.

Segundo o censo 2010 do Instituto Brasileiro de Geografia e Estatística o Brasil possuía aproximadamente 30 milhões de pessoas residindo em área rural. Segundo Dedecca (2013) grande parte deste total sofre de carências econômicas e sociais, vivendo em situação de pobreza. $\mathrm{O}$ autor destaca que a configuração atual da pobreza rural deve ser entendida como parte das transformações estruturais na atividade agrícola com efeitos sociais diferenciados no território nacional.

A região Sul do Brasil concentra aproximadamente 15\% de toda a população brasileira, é de grande importância pela sua participação na produção industrial, na geração de empregos e principalmente pela sua representatividade na agricultura. O setor agropecuário da região do Sul está suscetível às mesmas condições observadas em outras localidades, como clima, mercado e concorrência internacional. Tais fatores podem contribuir para queda da renda na agricultura e levar muitos trabalhadores e produtores a situação de miséria.

Destacar onde estão situados os municípios com elevado percentual de pobres e identificar se existem possíveis agrupamentos é de suma importância, nesse sentido, a utilização de mapas tem permitido obsevar de forma mais detalhada onde estão localizados os municípios com taxa de pobreza acima da média, tal informação ajuda na elaboração de políticas públicas mais focadas em busca da solução para tal problema.

Neste contexto o objetivo do presente artigo é verificar se existe relação espacial entre a taxa de pobreza o percentual da população rural nos municípios da região Sul do Brasil nos anos de 2000 e 2010. Além dessa breve introdução o trabalho apresenta o referencial teórico sobre o tema, à metodologia usada na pesquisa, os resultados encontrados e a conclusão.

\section{REFERENCIAL TEÓRICO E EMPÍRICO SOBRE POBREZA}


Sen (2001) destaca que a pobreza não é somente falta de renda, mas também envolve privações de capacidades fundamentais que não estão inseridos no seu conjunto orçamentário e outras formas de riqueza ou renda, podendo envolver características pessoais e características externas. Logo, diferentes pessoas podem apresentar diferentes necessidades, mas envolve dimensões econômicas e não econômicas.

Segundo Silva e Barros (2006), as contribuições de Amartya Sen, Anand e Dasgupta têm orientado a construção de indicadores de pobreza multidimensionais, que introduzem a noção de quais as dimensões relevantes, as variáveis adotadas e seus pesos, o método de agregação da pobreza e a forma de agregar a pobreza de todas as pessoas, de foram a analisar o desenvolvimento humano, de foram a construir um indicador escalar sintético de pobreza.

A noção de pobreza conceitual e operacionalmente relevante depende das condições de vida vigentes e do nível de desenvolvimento do sistema estatístico em cada sociedade. A pobreza é um fenômeno multidimensional, logo envolve insuficiência de renda, desigualdade na distribuição da renda produzida socialmente, restrição ao acesso a serviços básicos, à informação, ao trabalho e renda digna participação, entre outros fatores (ROCHA, 1998).

Para Rocha (2006), as linhas de pobreza expressam o número de indigentes e está relacionada à pobreza absoluta, que considera a necessidade de sobrevivência física e o não atendimento das necessidades nutricionais mínimas essenciais a sobrevivência humana, enquanto a pobreza relativa associa-se ao número de pobres de um país e também inclui além das necessidades alimentares, outras necessidades básicas a exemplo da habitação e do vestuário, por exemplo, e devem ser satisfeitas de forma a reduzir as desigualdades existentes no meio social.

O conceito de pobreza absoluta é importante no Brasil porque há muitas pessoas que não têm suas necessidades básicas, nutricionais e biológicas atendidas. As linhas de pobreza adotadas no Brasil, que apresenta uma economia bem monetizada, adotam os valores do salário mínimo ou seus múltiplos (MOCELIN, 2010).

O meio rural, segundo Schneider e Fialho (2000), passou por transformações na década de 70, mas nas análises de desenvolvimento dualistas sempre foi considerado mais atrasado e menos desenvolvido que as regiões urbanas. As transformações agrícolas no Brasil na década de 70 aprofundaram a desigualdade e a exclusão social da população rural. Segundo Rocha (2006), a pobreza rural no Brasil em 1999 era de 22,6\%, enquanto a pobreza no meio urbano era de $19,0 \%$ e nas regiões metropolitanas era de $23,7 \%$. 
Níger e Silva (2004) mostram que no período de 1995 e 2001 a pobreza nas áreas rurais apresentou queda significativa, com destaque para os estados do Ceará, Rio de Janeiro, Santa Catarina e Goiás enquanto para o estado de São Paulo observou-se uma elevação significativa da pobreza nas áreas rurais.

Em seu trabalho sobre fonte de renda rural não agrícola no Brasil Kageyama (2001) mostrou que as rendas de domicílios agrícolas no Brasil são bastante heterogêneas. A renda domiciliar per capita média de uma família agrícola na região Nordeste ampliada (incluindo Tocantins e Mato Grosso) equivale a um terço daquela observada na região mais rica São Paulo, Mato Grosso do Sul e Minas Gerais.

A proporção de pobres é muito maior entre as famílias cujo chefe tem na agricultura sua atividade principal e que possuem domicílio rural, mesmo os que se concentram nas periferias das pequenas e médias cidades, constituindo verdadeiras favelas rurais, estão relativamente em melhor situação que aqueles que moram na zona rural. E dentre os que moram no campo, os que estão ocupados em atividades não agrícolas vivem em melhor situação que os pequenos produtores e trabalhadores rurais temporários (SILVA, 1998).

Para Helfand e Pereira (2012) a pobreza rural no Brasil diminuiu consideravelmente nos últimas décadas, mas continua a ser um problema sério e um foco importante das políticas públicas. Grande parte do declínio é atribuída às inovações das políticas de seguridade social e de transferências de renda condicionada, tendo também o plano real sido de grande importância.

Os trabalhos que utilizam a Análise Exploratória dos Dados Espaciais a nível municipal têm crescido no Brasil, em partes pela disponibilidade de dados desagregados e pelo desenvolvimento de softwares destinados a este fim. No que tange a região Sul Cancian, Vidigal e Vidigal (2013) analisaram a pobreza nos municípios na região Sul para os anos de 1991 e de 2000, encontraram evidências de relação espacial da pobreza e da desigualdade nos municípios, o I de Moran foi positivo em ambos os anos analisados.

Teixeira (2014) analisou a distribuição espacial da pobreza nos municípios alagoanos. Utilizou a técnica de análise exploratória de dados espaciais e os modelos de econometria espacial. Os resultados obtidos mostraram haver forte discrepância entre as mesorregiões do estado, principalmente entre as mesorregiões Leste e Sertão, relação inversa entre a pobreza e a variável renda per capita e taxa de urbanização, e o contrário com relação ao índice de Gini.

Paula, Dalberto e Bohn (2013) estudaram a pobreza rural utilizando análise exploratória de dados espaciais. Os resultados alcançados indicam que a proximidade espacial influencia na 
concentração de determinadas características que tornam uma região mais pobre comparada a outras.

\subsection{População rural e taxa de pobreza nos estados brasileiros em 2000 e 2010: Evidências empíricas}

Na Tabela 1 é possível observar que entre 2000 e 2010 houve uma variação negativa de $3 \%$ da população rural Brasileira, a qual passou de $19 \%$ para $16 \%$. Na região Centro-Oeste o percentual de população rural era de $13 \%$ no ano de 2000, para o ano de 2010 a taxa percentual diminuiu para $11 \%$, considerando o período de analise houve variação negativa de $2 \%$. As regiões Norte e Nordeste concentram grande parte de suas populações morando em áreas rurais. No ano de 2000 aproximadamente $30 \%$ da população moravam nas áreas rurais dessas regiões. Já no ano e 2010 no norte aproximadamente a população rural representava 26\% e no nordeste em torno de $27 \%$, variação de aproximadamente 4 pontos percentuais negativo. As regiões sul e sudeste apresentam os menores percentuais de sua população morando em áreas rurais, tanto no ano de 2000 como de 2010. Na região sul da população total, 19\% moravam em áreas rurais, para o ano de 2010 o percentual diminuiu para 15\%. A região Sudeste apresenta o menor percentual de população residente em área rural, aproximadamente 9,5\% no ano de 2000 e no ano de 2010 o percentual diminuiu para $7 \%$, queda de 2,5 pontos percentuais.

Tabela 1 - Percentual da população rural nos estados da região sul do país em 2000 e 2010.

\begin{tabular}{lrrr}
\hline & $\mathbf{2 0 0 0}$ & $\mathbf{2 0 1 0}$ & Variação \\
\hline Brasil & 19 & 16 & -3 \\
Centro-Oeste & 13 & 11 & -2 \\
Nordeste & 31 & 27 & -4 \\
Norte & 30 & 26 & -4 \\
Sudeste & 9,5 & 7,0 & $-2,5$ \\
Sul & 19 & 15 & -4 \\
\hline
\end{tabular}

Fonte: IBGE.

2.2 População rural e taxa de pobreza nos estados do Sul do Brasil em 2000 e 2010: Evidências empíricas

Na Tabela 2, no ano de 2000 os estados da Região Sul que apresentou maior percentual de pessoas residindo em áreas rurais da foi Santa Catarina com 21\%, seguido por Paraná 19\% e Rio grande do Sul com 18\%. No ano de 2010 houve uma diminuição percentual de população 
rural nos estados desta região, novamente Santa Catarina apresentou a maior percentagem com $16 \%$ e o Paraná e Rio Grande do Sul tiveram o mesmo percentual de $15 \%$.

É possível destacar ainda que no ao de 2010 existiu uma redução da taxa populacional de moradores na áreas rurais se comparado com o ano de 2000 para todas as regiões do Brasil, seguindo uma tendência de aumento da urbanização.

Tabela 2 - Percentual de população rural nos estados da região Sul do Brasil em 2000 e 2010.

\begin{tabular}{lrrr}
\hline Estados & $\mathbf{2 0 0 0}$ & $\mathbf{2 0 1 0}$ & Variação \\
\hline Santa Catarina & 21 & 16 & -5 \\
Paraná & 19 & 15 & -4 \\
Rio Grande do Sul & 18 & 15 & -3 \\
\hline
\end{tabular}
Fonte: IBGE.

Na Tabela 3 é possivel destacar que na Região Sul do Brasul, o estado que apresentou a maior taxa de pobreza foi a Paraná, para ambos os anos, mas tambem que sua redução relativa, foi superior a dos demais estados desta região. No ano de 2000 os estados com maiores taxas de pobreza alem do Parana com 19\%, seguido pelo Rio Grande do Sul 16\% e Santa catarina com $13 \%$.

Para o ano de 2010 ocorreu uma redução na taxa de pobreza em todos os estados da região. No entando os estados do Parana e Rio Grande do Sul, apresentam as maiores taxas de pobreza.

Tabela 3 - Percentual da taxa de pobreza nos estados da região Sul do Brasil em 2000 e 2010.

\begin{tabular}{|c|c|c|c|}
\hline Estados & 2000 & 2010 & Variação \\
\hline Paraná & 19 & 6 & -13 \\
\hline Rio Grande do Sul & 16 & 6 & -10 \\
\hline Santa Catarina & 13 & 4 & -9 \\
\hline
\end{tabular}
Fonte: IPEA.

\section{PROCEDIMENTOS METODOLÓGICOS}

Nesta seção é abordada a metodologia utilizada na pesquisa, primeiramente são apresentados os elementos que compõem a análise exploratória de dados espaciais e posteriormente os correspondentes bancos de dados.

\section{ANÁLISE EXPLORATÓRIA DE DADOS ESPACIAIS}


Ao trabalhar com dados espaciais Almeida (2012), Diniz (2012), Medeiros et al. (2013) destacam que a analise exploratória de dados espaciais é o método mais adequado. Segundo Perobelli et al. (2007) a análise exploratória de dados espaciais é a técnica para descrever e visualizar distribuições espaciais, identificar localidades espaciais atípicas, descobrir padrões de associação espacial e sugerir diferentes regimes espaciais.

O primeiro passo no estudo de AEDE é testar a hipótese de que os dados espaciais sejam distribuídos aleatoriamente. Intuitivamente, aleatoriedade espacial significa que os valores de um atributo numa região não dependem dos valores deste atributo nas regiões vizinhas (ALMEIDA, 2012).

No entanto essa análise é mais apropriada na investigação de variáveis espacialmente densas ou intensivas, ou seja, variáveis que são divididas por algum indicador de intensidade (variáveis per capita, por área, etc.). Nesse sentido Perobelli et al. (2007) reforça que a partir da AEDE é possível extrair medidas de autocorrelação espacial global e local, investigando a influência dos efeitos espaciais por intermédio de métodos quantitativos. Entretanto, é necessário anteriormente definir uma matriz de pesos espaciais a fim de determinar o grau de interação entre as regiões.

\section{MATRIZES DE PESOS ESPACIAIS}

O conceito de matrizes de pesos espaciais é baseado na contiguidade, que, por sua vez, pode ser definida de acordo com a vizinhança, a distância tanto geográfica quanto socioeconômica, bem como um a combinação disso. Vários resultados em econometria espacial são sensíveis à escolha da matriz de pesos espaciais. Portanto, a discussão a respeito da tipologia das matrizes é questão importante e delicada na literatura (ALMEIDA, 2012). Das formas de matrizes de pesos espaciais utilizadas, as mais comuns são a convenção rainha e torre apresentadas na figura 1.

Figura 1 - Matrizes de contiguidade

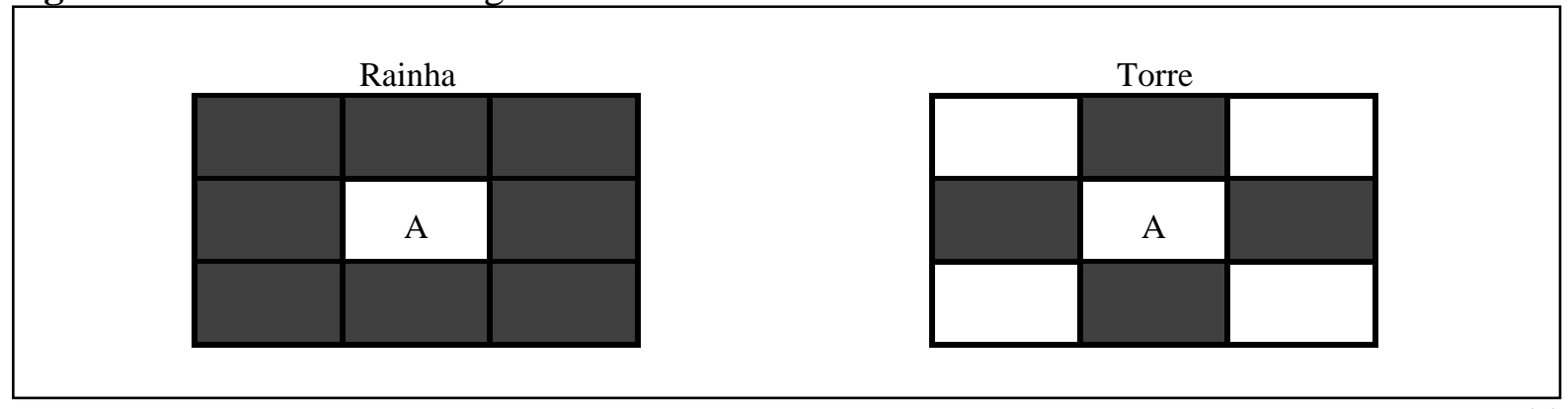


Fonte: Elaborado pelos autores com base em Almeida (2012).

A convenção de contiguidade é dita ser rainha, caso além das fronteiras com extensão diferente de zero, puderem ser considerados os vértices (nós), na visualização de um mapa, como contíguos. Caso apenas as fronteiras físicas com extensão diferente de zero entre as regiões sejam levadas em conta, a convenção de contiguidade é considerada como torre. Essas duas convenções são as mais utilizadas na literatura.

\section{A ESTATÍSTICA I DE MORAN UNIVARIADA}

Segundo Almeida (2012) a estatística I de Moran é um coeficiente de autocorrelação espacial, usando a medida de autocovariância na forma de produto cruzado. Foi proposta por Patrick A. P. Moran no ano de 1948, sendo considerado o primeiro coeficiente de autocorrelação espacial. A notação matricial da estatística I de Moran é representada pela equação 1:

$$
\mathrm{I}=\frac{\mathrm{n}}{\mathrm{S}_{0}} \frac{\mathrm{z}^{\prime} \mathrm{Wz}}{\mathrm{z}^{\prime} \mathrm{z}}
$$

Em que $n$ é o número de regiões, $z$ denota os valores da variável de interesse padronizada, $W z$ representa os valores médios da variável de interesse padronizada nos vizinhos, definidos segundo uma matriz de ponderação espacial $W$. Um elemento dessa matriz, referente à região $i$ e a região $j$, é registrado como $w_{i j}$, significando que todos os elementos da matriz de pesos espaciais $w$ devem ser somados.

Os valores de I maiores (ou menores) do que o valor esperado de $E(I)=[-1 /(n-1)]$ significa que há autocorrelação positiva (ou negativa). De acordo com Almeida (2012), a autocorrelação espacial positiva revela que existe uma similaridade entre os valores do atributo estudado e da localização espacial do atributo. A autocorrelação espacial negativa revela, por sua vez, que existe uma dissimilaridade entre os valores do atributo considerado e a localização espacial.

Segundo Almeida (2012) o I de Moran fornece três tipos de informações: 1) o nível de significância fornece a informação sobre os dados estarem distribuídos aleatoriamente ou não; 
2) o sinal positivo da estatística I de Moran, desde que significativos, indica que os dados estão concentrados em regiões. O sinal negativo, por sua vez, indica a dispersão dos dados; 3) a magnitude da estatística fornece a força da autocorrelação espacial, quanto mais próximo de um mais forte é autocorrelação e quanto mais próximo de -1 mais disperso estão os dados.

\section{O DIAGRAMA DE DISPERSÃO DE MORAN UNIVARIADO}

O diagrama de dispersão de Moran é exibido na Figura 2, segundo Almeida (2012) o diagrama de dispersão de Moran univariado é uma alternativa para visualizar a autocorrelação espacial, o valor da variável de interesse é colocado no eixo horizontal $(x)$ e a defasagem espacial da variável de interesse no eixo vertical $(y)$.

De acordo com Diniz (2012), além da medida global de associação linear espacial, o diagrama dispersão mostra associação espacial entre as regiões e seus vizinhos dividido em quatro quadrantes: alto-alto (AA), baixo-baixo (BB), alto-baixo (AB) e baixo-alto (BA).

Quando o I de Moran é positivo a reta de regressão apresenta inclinação ascendente e os dados tendem a estar agrupado no primeiro e terceiro quadrante, porém quando o I de Moran é negativo a reta de regressão é descendente e dados aparecem localizados em sua grande maioria no segundo e quarto quadrante (figura 2).

Figura 2 - Diagrama de dispersão de Moran Univariado

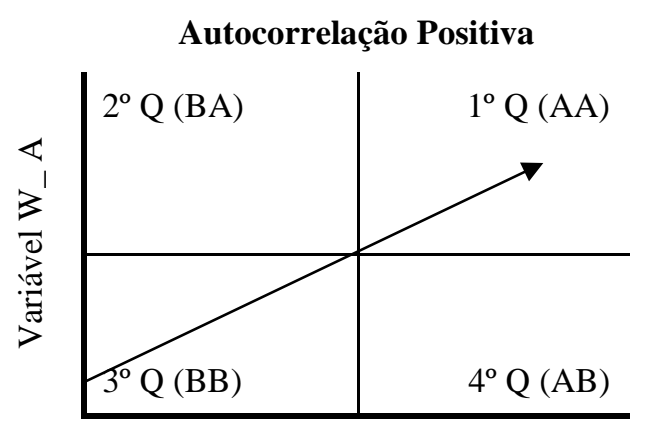

Variável A

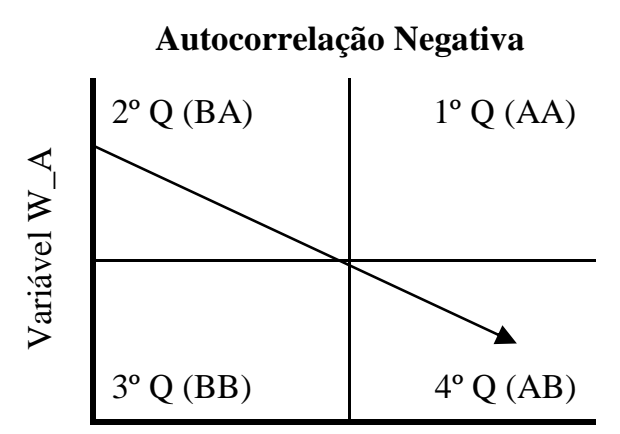

Variável A

Fonte: Elaborado pelos autores.

Um agrupamento alto-alto (AA) significa que as unidades espaciais pertencentes a esse agrupamento exibem valores altos da variável de interesse rodeados por unidades espaciais que apresentam valores também altos, representado pelo primeiro quadrante do diagrama. Um agrupamento baixo-baixo (BB) refere-se a um agrupamento cujas unidades espaciais mostram 
valores baixos circundados por unidades espaciais que ostentam valores também baixos, representado pelo terceiro quadrante.

Um agrupamento alto-baixo $(\mathrm{AB})$ diz respeito a um cluster no qual uma unidade espacial qualquer com um alto valor da variável de interesse são circunvizinhos de unidades espaciais com um baixo valor. Isso é representado pelo quarto quadrante. Um agrupamento baixo-alto (BA) concerne a um cluster no qual uma unidade espacial qualquer com um baixo valor da variável de interesse são circundados por unidades espaciais com alto valor, essa situação é representada no segundo quadrante.

\section{INDICADOR LOCAL DE ASSOCIAÇÃO ESPACIAL (LISA)}

O indicador LISA demonstra o grau de autocorrelação espacial local, de acordo com Anselin (1995) para que isso ocorra é necessário que essa estatística satisfaça a dois critérios: a) esses indicadores devem possuir, para cada observação, uma indicação de clusters espaciais significantes de valores similares ao redor de cada observação, b) o somatório dos indicadores LISA, em todas as regiões, deve ser proporcional ao indicador de autocorrelação espacial global. Dessa maneira, os indicadores LISA podem ser representados por intermédio da equação 2:

$$
I_{i, t}=\frac{\left(X_{i, t}-\mu\right)}{M_{0}} \sum_{j} W_{i, j}\left(X_{i, j}-\mu_{t}\right) \quad \text { Em que é } M_{0}=\frac{\left(X_{i, t}-\mu_{t}\right)^{2}}{n}
$$

Na qual $X_{i, j}$ é a observação e uma variável de interesse na região $i$ para o ano $t, \mu_{t}$ é a média das observações entre as regiões para o ano $t$, no qual o somatório em relação à $j$ é tal que somente os valores vizinhos de $j$ são incluídos.

De acordo com Anselin (1995), a estatística LISA, é usada para testar a hipótese nula, ou seja, a ausência de associação espacial local. Assim, deve-se fazer uso de uma aleatorização condicional, que permitia determinar pseudoníveis de significância.

Para a obtenção de uma distribuição empírica das estatísticas de teste, deve-se observar se o valor da variável de interesse está dentro ou fora da região critica definida. Dessa maneira, se o valor calculado for superior em magnitude à esperança matemática do I de Moran, seus resultados serão estatisticamente significativos. 


\section{ESTATISTICA I DE MORAN GLOBAL BIVARIADA}

No estudo da Análise Exploratória dos Dados Espaciais (AEDE) é possível obter um coeficiente de autocorrelação espacial global bivariado, o objetivo é descobrir se o valor de um atributo observado numa dada região esta relacionado espacialmente com os valores de outra variável observada em regiões vizinhas (ALMEIDA, 2012). Assim é possível calcular a estatística I de Moran para duas varáveis diferentes padronizadas, digamos, $\mathrm{Z}_{1}$ e $\mathrm{Z}_{2}$. Somente os vizinhos da observação $i$, definidos conforme uma matriz de pesos espaciais é incluída no cálculo. E se a matriz (W) for normalizada na linha, a equação é apresentada na sua forma matricial conforme a equação 3 :

$$
\mathrm{I}^{\mathrm{Z}_{1} \mathrm{Z}_{2}}=\frac{\mathrm{Z}_{1}^{\prime}}{\mathrm{Z}_{1}^{\prime}} \frac{\mathrm{W}_{\mathrm{z2}}}{\mathrm{Z}_{1}}
$$

Em que $\mathrm{WZ}_{2}$ é a defasagem espacial da variável padronizada $\mathrm{Z}_{2}$. Este coeficiente tem dois componentes distintos, como se trata da versão bivariada da estatística $I$ de Moran, o numerador refere-se a uma medida de covariância do tipo, produto cruzado e o denominador diz respeito a um reescalonamento, usando a variância de dados. De acordo com Anselin et al. (2003) essa estatística dá uma indicação do grau de associação linear (positiva ou negativa) entre o valor para uma variável em uma dada locação $i$ e a média de uma outra variável nas locações vizinhas $j$.

\section{DIAGRAMA DE DISPERSÃO DE MORAN BIVARIADO}

O diagrama de Moran bivariado permite verificar a relação linear entre duas variáveis e possibilita observar a distribuição em quatro associações espaciais: alto-alto (AA), baixo-baixo $(\mathrm{BB})$, alto-baixo $(\mathrm{AB})$ e baixo-alto $(\mathrm{BA})$. A variável $(\mathrm{A})$ de interesse é plotada no eixo $(x)$, ao passo que a outra variável de interesse defasada (W_B) é colocada no eixo (y), quando a relação é positiva a inclinação da reta é ascendente e os dados tendem a agrupar-se em sua grande maioria no primeiro e terceiro quadrante. Entretanto, quando a relação entre duas variáveis é negativa a inclinação da reta é descendente e as regiões estudadas tendem a se concentrar no segundo e no quarto quadrante (figura 3). 
Figura 3 - Diagrama de Dispersão de Moran Bivariado

Autocorrelação positiva

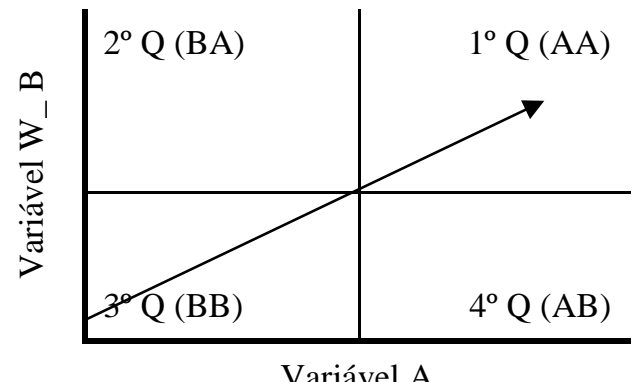

Variável A
Autocorrelação negativa

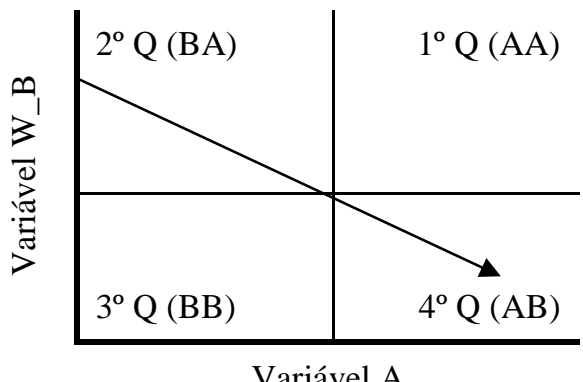

Variável A

Fonte: elaborado pelos autores.

O regime alto-alto (AA) representa as associações espaciais que exibem valor elevado da variável $(A)$ e são cercados por municípios com elevado valor da variável defasada $\left(W_{-} B\right)$, representados pelo primeiro quadrante. O agrupamento (BA) representa as localidades espaciais que apresentam baixo valor da variável de interesse $(A)$ e são vizinhos de municípios que exibem alto valor da variável $\left(W_{-} B\right)$, exibidas no segundo quadrante.

Um agrupamento baixo-baixo (BB) é formado por unidades espaciais que contemplam baixo valor da variável $(A)$ e são cercadas por baixo valores da variável defasada $\left(W_{-} B\right)$. Um agrupamento alto-baixo $(\mathrm{AB})$ representa as unidades municipais com alto valor da variável $(A)$ e são cercados por municípios com baixo valor de $\left(W_{-} B\right)$.

\section{ESTATISTICA I DE MORAN LOCAL BIVARIADA}

Segundo Almeida (2012) pode ser obtido um coeficiente de autocorrelação espacial local num contexto multivariado, o I de Moran local bivariado é calculado de acordo com a equação 4:

$\mathrm{Ii}^{\mathrm{z} 1 \mathrm{z2}}=\mathrm{Z}_{1 \mathrm{i}} \mathrm{WZ}_{2 \mathrm{i}}$

Em que $w_{2} z_{2 i}$ é a defasagem espacial da variável padronizada $z_{2 i}$. Semelhantemente à fórmula do calculo do I de Moran na equação 3, somente os vizinhos da observação $i$, definidos conforme uma matriz de pesos espaciais, são incluídos no cálculo. 
De acordo com Anselin et al. (2003), essa estatística dá uma indicação do grau de associação linear (positiva ou negativa) entre o valor para uma variável em uma dada região $(i)$ e a média de uma outra variável nas localidades vizinhas (j). É possível mapear os valores da probabilidade da medida, estatisticamente significativos, gerando o chamado mapa de significância bivariado do Moran local.

\section{BASE DE DADOS}

A base de dados esta disponível no site do Instituto Brasileiro de Geografia e Estatística (IBGE), censos de 2000 e 2010 e do Instituto de Pesquisa Economia Aplicada (IPEA). Os dados utilizados são: percentual da população rural e taxa de pobreza dos municípios brasileiros de 2000 e 2010.

Para mensurar o percentual da população rural utilizou-se a divisão simples da população rural pela população total multiplicado por 100. Para a variável taxa de pobreza, utilizou-se proporção dos indivíduos com renda domiciliar per capita igual ou inferior a $\mathrm{R} \$ 140,00$ mensais, em reais de agosto de 2010 para os municípios brasileiros. O universo de indivíduos é limitado àqueles que vivem em domicílios particulares permanentes.

\section{ANÁLISE DOS RESULTADOS}

Nesse item é realizada a análise exploratória de dados espaciais das seguintes variáveis: percentual da população rural e taxa de pobreza nos municípios da região Sul do país.

\section{MAPA DE DESVIO PADRÃO}

Na Figura 4 é apresentada a distribuição espacial da taxa de pobreza dos municípios da Região Sul para os anos de 2000 e de 2010 utilizando o mapa de desvio-padrão. O referido mapa permite verificar onde estão situados os municípios que contemplam taxa de pobreza acima e abaixo da média, tal critério possibilita identificar a quantidade de unidades espaciais com um desvio padrão, com dois desvios padrão e também a presença de outliers alto e baixo. Os outliers indicam quantos e onde estão localizados os municípios no ano de 2000 e de 2010 com taxa de pobreza muito acima ou abaixo da média. 
Figura 4 - Mapa de desvio padrão da taxa de pobreza de 2000 e 2010
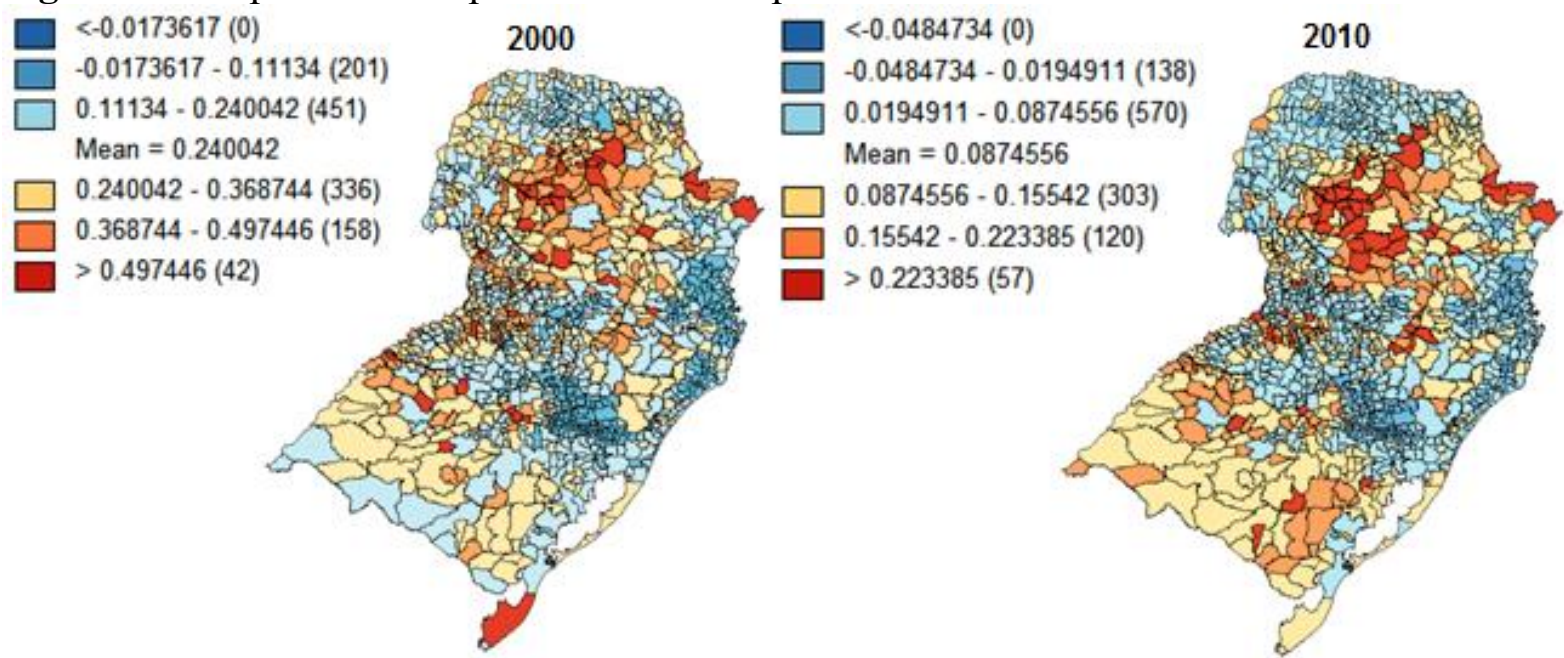

Fonte: Elaborado pelos autores com dados do IBGE e utilização do Software Geoda

$\mathrm{Na}$ Figura 4, pode se observar no mapa de desvio padrão da taxa de pobreza no ano de 2000, que 38\% dos municípios do Sul apresentaram até um desvio padrão acima da média, 17\% entre um e dois desvio padrão acima da média e não foram observados outlier. Dos municípios que se situaram abaixo da média, 28\% apresentam até um desvio padrão, 13\% apresentaram entre um e dois desvio padrão e $4 \%$ foram outliers.

No ano de 2010 houve um pequeno acréscimo de municípios que ficaram acima da média, do total 48\% dos municípios estavam a um desvio padrão acima da média, 12\% entre um e dois desvio padrão e também não houve nenhum outlier, em relação aos municípios abaixo da média, 25\% apresentaram até um desvio padrão, 10\% apresentaram entre um e dois desvio padrão e $5 \%$ foram outliers.

\section{DE MORAN GLOBAL UNIVARIADA}

A Tabela 4 indica os valores I de Moran para a variável taxa de pobreza da região Sul no ano de 2000 e de 2010, foi utilizada uma matriz de pesos espaciais do tipo Rainha (Queen). Como pode ser verificado, existe autocorrelação espacial positiva da variável a um nível de significância de $1 \%$.

Tabela 4 - Autocorrelação global univariada

\begin{tabular}{cccc}
\hline Variável & Convenção & I de Moran & Probabilidade \\
\hline TX_POB 2000 & Rainha $($ QUEEN) & 0,5959 & 0,001 \\
\hline
\end{tabular}




\begin{tabular}{ccc}
\hline TX_POB 2010 & 0,5708 & 0,001 \\
\hline Fonte: Elaborado pelos autores com dados do IBGE.
\end{tabular}

Fonte: Elaborado pelos autores com dados do IBGE.

O sinal positivo da estatística I de Moran da Tabela 4 indica que os municípios que apresentam taxa de pobreza elevada estão cercados por unidades municipais com taxa de pobreza acima da média e localidades municipais que exibem taxa de pobreza abaixo da média são circunvizinhados por municípios com baixa taxa de pobreza. Verifica-se, que entre o período de 2000 e 2010 não houve alteração significativa da associação espacial, apenas uma pequena redução, ou seja, tem-se quase a mesma concentração espacial da taxa de pobreza nos municípios, sugerindo quase a mesma concentração espacial do I de Moran nos dois anos.

\section{DIAGRAMA DE DISPERSÃO DE MORAN UNIVARIADO}

O diagrama de dispersão de Moran exibido na Figura 5 permite verificar qual o padrão de concentração dos municípios pelos quadrantes. No eixo horizontal esta plotada a taxa de pobreza dos municípios da região Sul e no eixo vertical a defasagem da taxa de pobreza.

Figura 5 - Diagrama de Dispersão de Moran da taxa de pobreza para os anos de 2000 e 2010
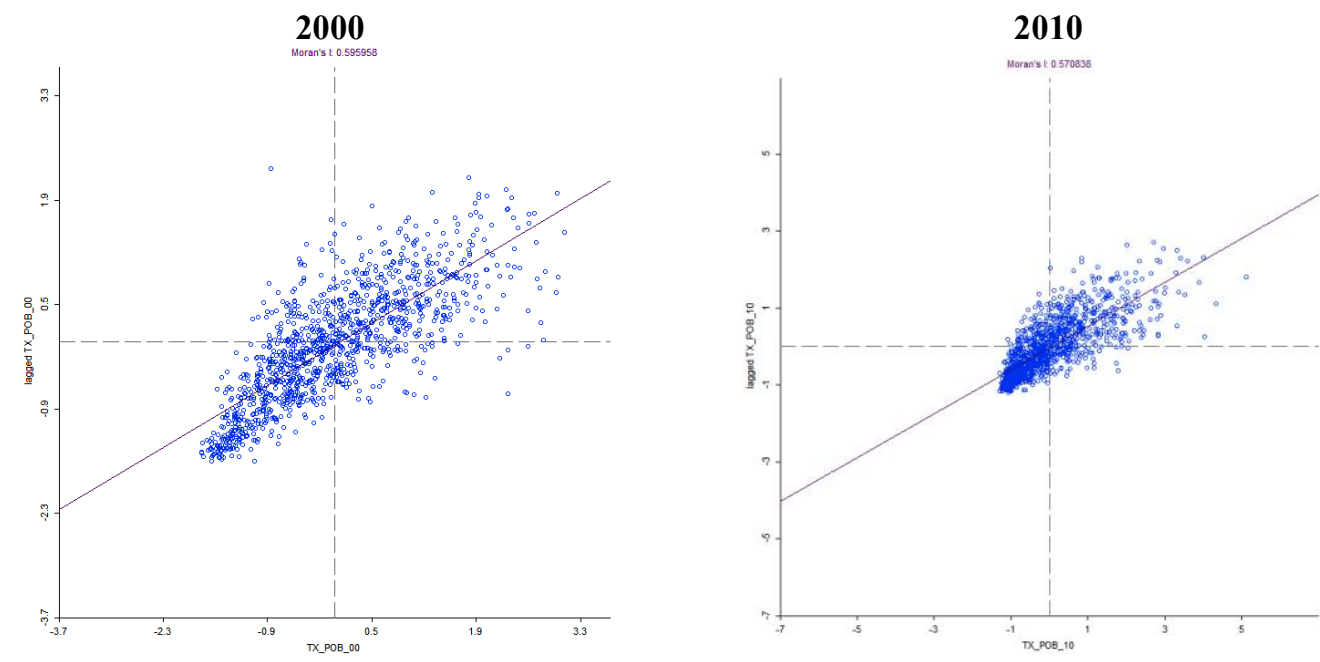

Fonte: Elaborado pelos autores com dados do IBGE e utilização do Software Geoda.

Ao observarmos a distribuição dos municípios no gráfico de Moran apresentado na Figura 5, foi verificado que no de 2000 em torno de $37 \%$ dos municípios analisados encontram-se no 
quadrante alto-alto (AA), aproximadamente $43 \%$ estão localizados no terceiro quadrante, ou seja, associação espacial baixo-baixo (BB). No que se refere às regiões atípicas, ou seja, aquelas que apresentam desvios em relação ao padrão global de autocorrelação se verificam que $11 \%$ dos municípios estão no quadrante baixo-alto (BA) e $9 \%$ no quadrante alto-baixo (AB).

No ano de 2010 foi verificado que 33\% dos municípios analisados estão no quadrante alto-alto (AA) em torno de $50 \%$ estão situados no terceiro quadrante, ou seja, regime espacial baixo-baixo (BB). No que tange as regiões atípicas, foi observado que $10 \%$ dos municípios se encontram no quadrante baixo-alto (BA) e 7\% estão concentrados no quarto quadrante, ou seja, são agrupamentos espaciais alto-baixo $(\mathrm{AB})$.

\section{MAPA DE SIGNIFICÂNCIA LISA E DE CLUSTER LOCAL UNIVARIADO}

Nas Figuras 6 são apresentados os mapas de significância e de cluster do ano 2000. No mapa de significância Lisa destacados em verde mais escuro estão os regimes estatisticamente significativos a $0,1 \%$ e os municípios destacados em verde mais claro as associações significativas a 5\%. Combinando as informações do diagrama de Moran (Figura 5) com o mapa de significância obtemos o mapa de cluster dividido em quatro categorias estatisticamente significantes, alto-alto, baixo-baixo, baixo-alto e alto-baixo.

Figura 6 - Mapa Lisa e de Cluster Taxa de Pobreza de 2000

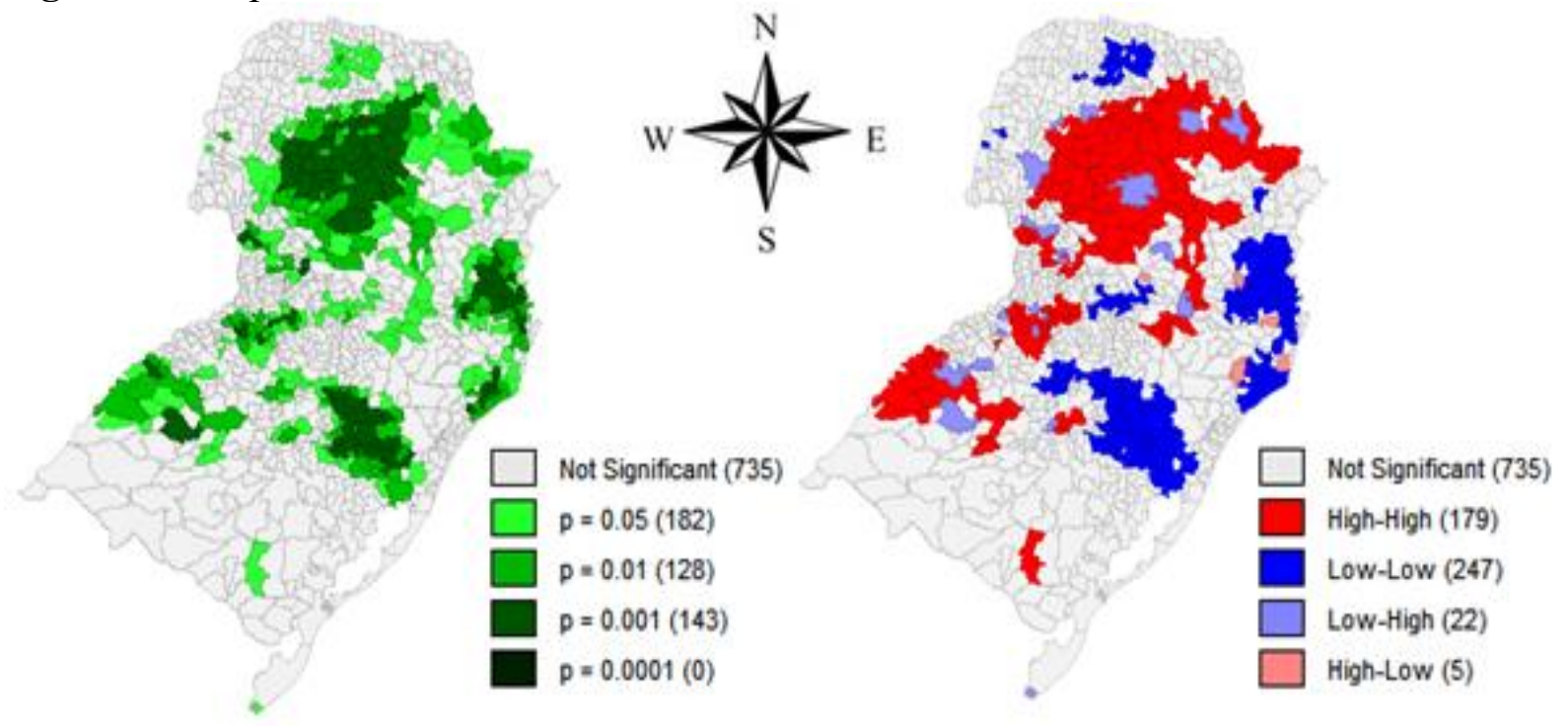

Fonte: Elaborado pelos autores com dados do IBGE e utilização do Software Geoda. 
No mapa de significância (Figura 6) foram observadas que 182 unidades municipais da região Sul apresentaram significância a $5 \%$, as associações espaciais significativas a $1 \%$ somaram 182 municípios e 143 apresentaram significância de $0,1 \%$.

No mapa de cluster do ano 2000 apresentado na Figura 6 pode ser observado à formação de associação espacial alto-alto (AA), as quais se concentraram nas regiões Sudoeste do Rio Grande do Sul, Centro-Sul e Sudeste Paranaense, esses regimes espaciais são formados por unidades municipais que exibem elevada taxa de pobreza e são cercados por vizinhos com elevada taxa de pobreza.

Por outro lado, o regime espacial baixo-baixo (BB) esta concentrado na região Metropolitana de Porto Alegre, no Vale do Itajaí, na grande Florianópolis e Norte Central do Paraná, demonstrando que nessas localidades os municípios exibem taxa de pobreza abaixo da média e são cercados por vizinhos com baixa taxa de pobreza.

Figura 7 - Mapa Lisa e de Cluster Univariado da taxa de pobreza de 2010

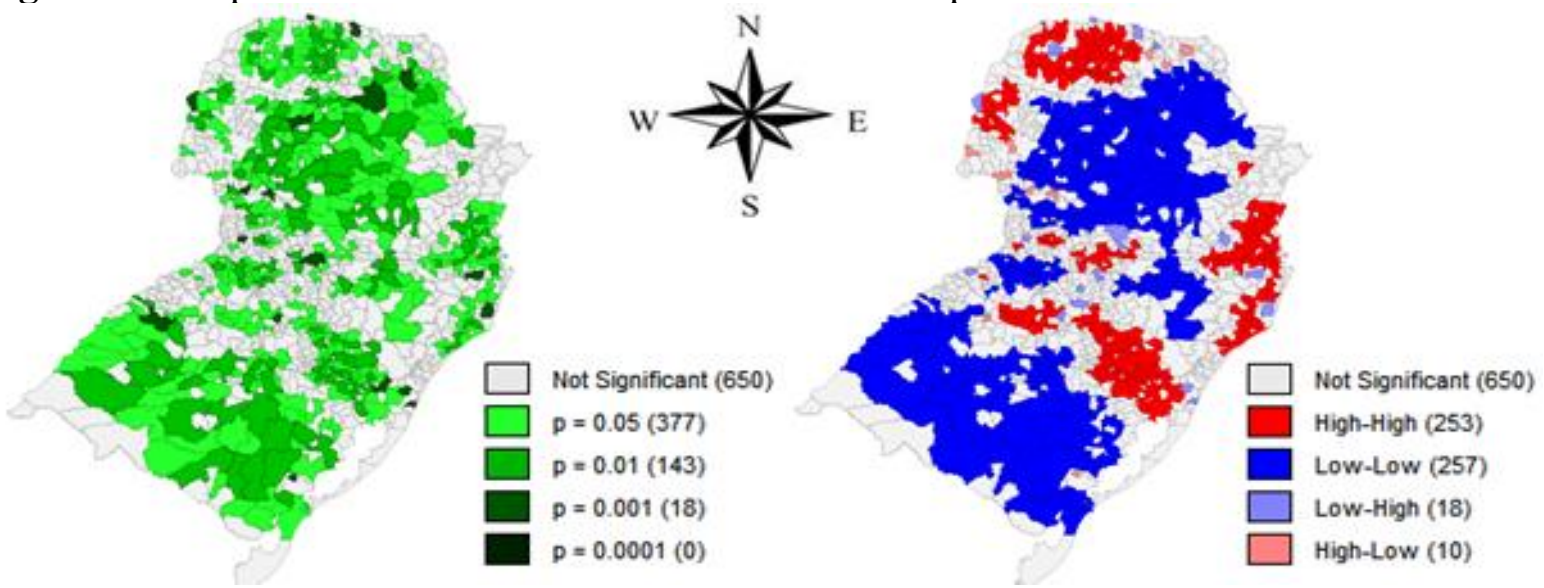

Fonte: Elaboração Própria com Dados do IBGE e Utilização do Software Geoda

Na Figura 7, observamos no mapa de significância da taxa de pobreza do ano de 2010 que 337 municípios apresentaram significância estatística a 5\%, outros 143 foram significativos a $1 \%$ e 18 unidades municipais foram significativas a $0,1 \%$.

O mapa de cluster do ano de 2010 é apresentado na Figura 7, estão em destaque as associações espacial alto-alto (AA) concentrado principalmente nas regiões Norte Central e Noroeste Paranaense, Vale do Itajaí, grande Florianópolis e Metropolitana de Porto Alegre, ou seja, são regiões que exibem taxa de pobreza abaixo da média cercados por vizinhos na mesma situação. 
O regime baixo-baixo (BB) aparece concentrado nas regiões Sudeste e Sudoeste do Rio Grande do Sul, no Centro-Sul, Sudeste, Norte Pioneiro e Centro Oriental Paranaense. Podemos destacar nos dois períodos, que municípios com alta taxa de pobreza, estão cercados por municípios também com altas taxas de pobreza, e os municípios com baixa taxa de pobreza estão cercados por municípios com a mesma característica.

\section{DE MORAN GLOBAL BIVARIADO}

Na Tabela 5 é possível observar o valor do I de Moran bivariado para as variáveis percentual de população rural e taxa de pobreza dos municípios da região Sul no ano de 2000 e de 2010. Foi utilizada uma matriz de pesos espaciais do tipo Rainha (Queen), o resultado indica a existência de autocorrelação espacial positiva entre as variáveis a um nível de significância de $1 \%$.

Tabela 5 - autocorrelação global bivariada

\begin{tabular}{c|c|c|c}
\hline Variável & Convenção & I de Moran & Probabilidade \\
\hline POP_RUR x TX_POB 2000 & Rainha & 0,1709 & 0,001 \\
POP_RUR x TX_POB 2010 & & 0,1751 & 0,001 \\
\hline
\end{tabular}

Fonte: elaborado pelos autores com dados do IBGE.

O sinal positivo da estatística I de Moran revela haver similaridade entre as variáveis analisadas, sendo assim, os municípios que contemplam taxa de pobreza acima da média são cercados por vizinhos com elevado percentual de população rural, e as unidades municipais onde a taxa de pobreza é baixa os municípios vizinhos tendem a ter mais pessoas residindo em áreas urbanas.

A estatística fornece o nível de associação espacial das variáveis nos dois períodos, ou seja, no ano de 2000 e de 2010 . Verifica-se, que praticamente não há alteração da associação espacial, tem-se quase a mesma concentração espacial entre o percentual da população rural e taxa de pobreza dos municípios, sugerindo não terem ocorrido mudanças significativas no período de uma década. 
Entretanto, conforme Haddad e Pimentel (2004) explicam que a análise da estatística I de Moran é adequada para verificar a existência de regimes espaciais, entretanto não torna possível a visualização do padrão de agrupamento dos dados e a sua evolução ao longo do tempo. Para isto os autores sugerem analisar o diagrama de dispersão de Moran.

\section{DIAGRAMA DE DISPERSÃO DE MORAN BIVARIADO}

O gráfico de dispersão de Moran permite verificar a distribuição da variável estudada considerando quatro associações espaciais, alto-alto (AA), baixo-baixo (BB), alto-baixo (AB) e baixo-alto (AB). No eixo das ordenadas esta plotado o percentual de população residindo em área rural e no eixo das abscissas a taxa de pobreza.

No primeiro quadrante esta o agrupamento com característica alto-alto, ou seja, exibem elevada de taxa de pobreza e são cercados por municípios com elevado percentual de pessoas residindo em área rural. No terceiro quadrante estão situados os municípios com baixa taxa de pobreza e possuem vizinhos com baixo percentual de pessoas morando em área rural.

Já no segundo quadrante estão localizadas as unidades municipais que contemplam baixa percentual de pessoas residindo em área rural, porém os vizinhos exibem elevada taxa de pobreza. No quarto quadrante estão concentrados os municípios que contemplam elevado percentual de morador rural, mas estão circunvizinhados por unidades espaciais com taxa de pobreza abaixo da média.

Figura 8 - Diagrama de dispersão de Moran bivariado da taxa de população rural e da taxa de pobreza para os anos de 2000 e 2010.
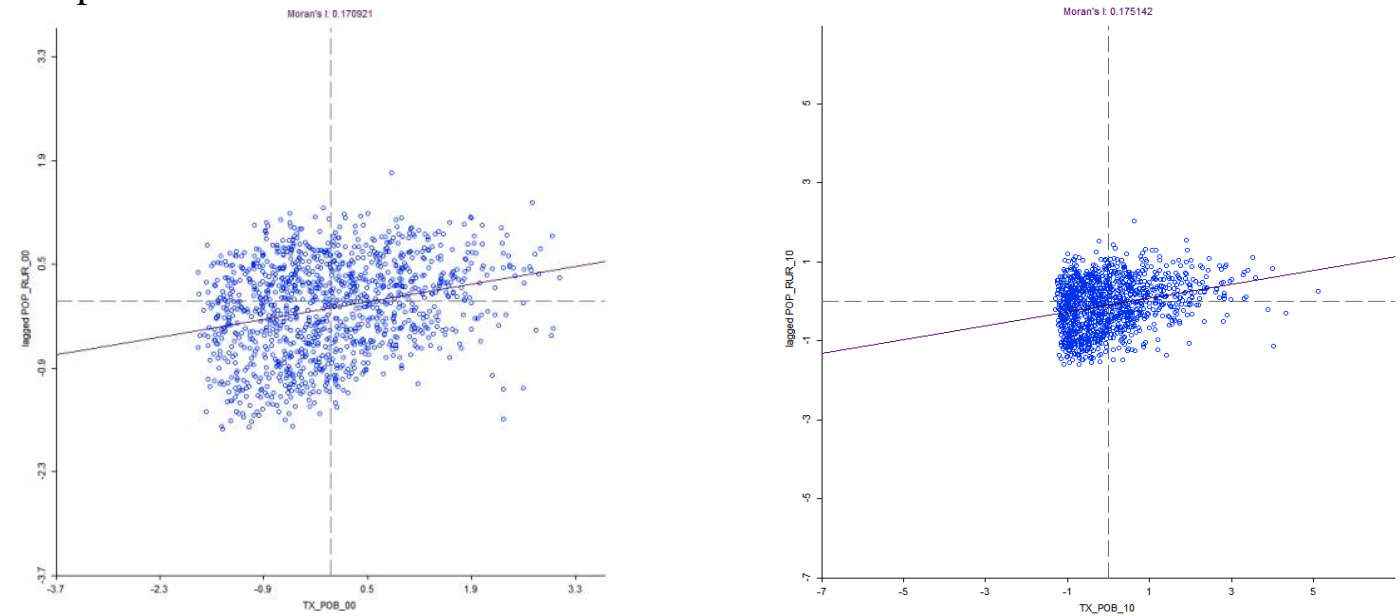

Fonte: Elaborado pelos autores com dados do IBGE e utilização do Software Geoda. 
Conforme apresentado na figura 8, foi possível verificar graficamente, a concentração espacial para a taxa de pobreza e o percentual da população rural dos municípios da região Sul nos anos de 2000 e de 2010. O resultado referente ao ano de 2000 mostra que $26 \%$ dos municípios analisados encontram-se no primeiro quadrante, ou seja, denotados com alto-alto (AA) e em torno de $33 \%$ estão situados no terceiro quadrante, sendo considerado, regime espacial baixo-baixo (BB). No que tange às regiões atípicas, ou seja, aquelas que apresentam desvios em relação ao padrão global de autocorrelação foram verificadas que $22 \%$ dos municípios estão localizados no segundo quadrante, classificadas com baixo-alto (BA) e aproximadamente $19 \%$ esta situada no quarto quadrante, assim, são agrupamento espacial altobaixo $(\mathrm{AB})$.

No gráfico do ano de 2010 em torno de $23 \%$ dos municípios analisados estão situados no primeiro quadrante, ou seja, alto-alto (AA) e aproximadamente $37 \%$ encontram-se localizados no terceiro quadrante, denotados assim, como baixo-baixo (BB). Nas regiões atípicas, representadas pelo segundo e quarto quadrante estão $40 \%$ dos municípios, sendo $24 \%$ denotados com baixo-alto (BA) e aproximadamente $16 \%$ regime espacial alto-baixo (AB).

\section{MAPA DE SIGNIFICÂNCIA LISA E DE CLUSTER LOCAL BIVARIADO}

É possível através de um mapeamento dos valores da probabilidade de medida estatisticamente significativos, gerar um mapa de significância bivariada do Moran Local. O mapa de cluster apresenta as quatro categorias estatisticamente significantes, combinando o mapa de significância local com o diagrama de dispersão de Moran (ALMEIDA, 2012).

O mapa de significância e de cluster que relaciona a taxa de pobreza e o percentual de população rural dos municípios da região Sul no ano 2000 é apresentado na figura 9 . No mapa lisa estão em destaque 191 unidades municipais apresentara significância estatística a 5\%, as quais estão denotadas em verde mais claro. Nos regimes espaciais estatisticamente significativos a $1 \%$ aparecem 141 localidades municipais e 38 apresentaram significância estatística a $0,1 \%$.

O mapa de cluster bivariado exibe as associações espaciais divididas em quatro categorias. A associação espacial alto-alto (AA) foi formada por 71 municípios, o que representara $21 \%$ do total de clusters significativos e se concentraram na região Central do Paraná e do Rio Grande do Sul. Por outro lado, o regime baixo-baixo (BB) é composto por 167 unidades municipais, ou seja, $49 \%$ das associações significativas, estas aparecem concentradas 
nas regiões do Norte do Paraná, no Litoral do paranaense e na região metropolitana de Santa Catarina e de Porto Alegre.

Figura 9 - Mapa Lisa e de Cluster Bivariado da População Rural e Taxa de Pobreza de 2000

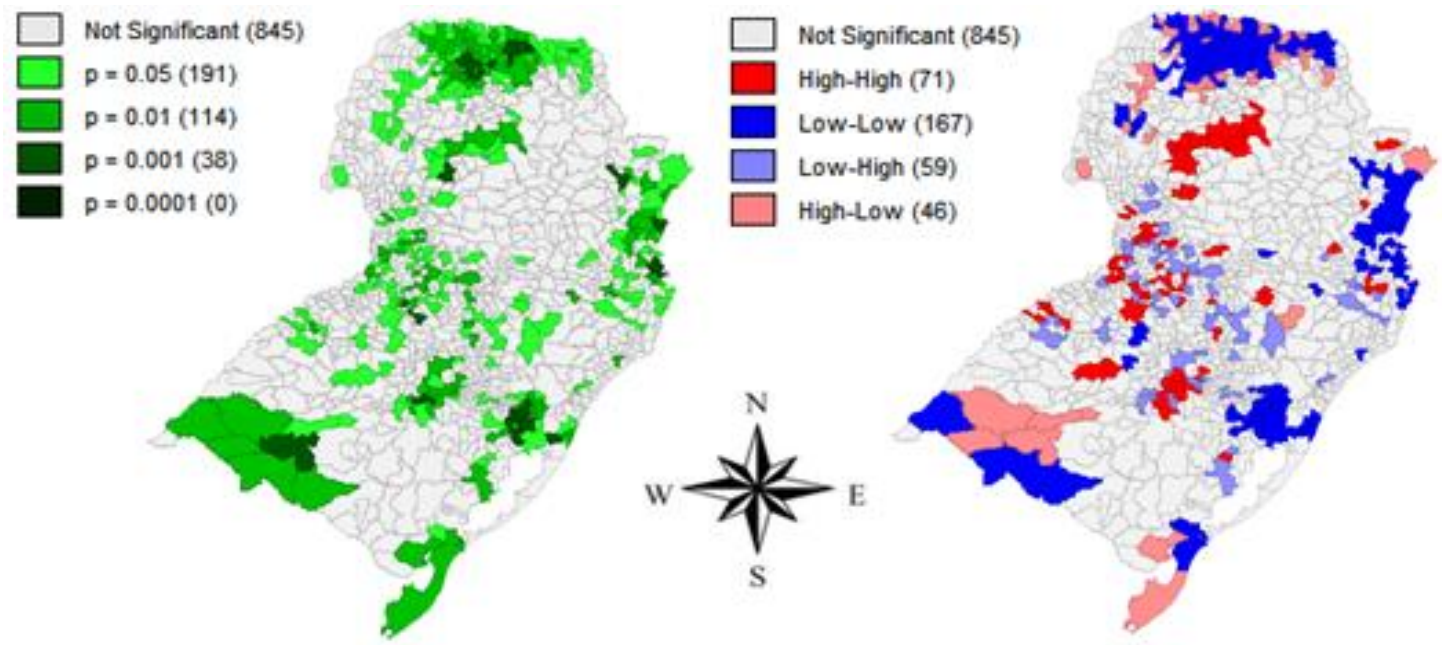

Fonte: Elaborado pelos autores com dados do IBGE e utilização do Software Geoda

A estatística I de Moran local para a população rural e taxa de pobreza no ano de 2010 é exibido na Figura 10. No mapa de significância é possível verificar que 188 unidades municipais apresentaram significância de 5\%, outras 121 localidades foram significativas a $1 \%$ em 40 municípios a significância foi de $0,1 \%$. Em comparação aos resultados observados para o ano de 2000 foi observado um leve queda dos municípios significativos a 5\% e um pequeno aumento das unidades com significância a $0,1 \%$ e $1 \%$

No mapa de cluster, ficou evidente que no ano de 2010, a formação de agrupamentos espaciais alto-alto (AA) continuou concentradas principalmente nas regiões Central do estado do Paraná e no Rio Grande do Sul, que são regiões com alto percentual de população rural com alta taxa de pobreza. O regime espacial baixo-baixo (BB) demonstra aglomerações de municípios que apresentaram baixo percentual de pessoas morando na área rural e com baixa taxa de pobreza, as mesmas foram identificadas no Norte paranaense, na região Metropolitana de Porto Alegre e na região litorânea de Santa Catarina.

Figura 10 - Mapa Lisa e de Cluster Bivariado da População Rural e Taxa de Pobreza de 2010 


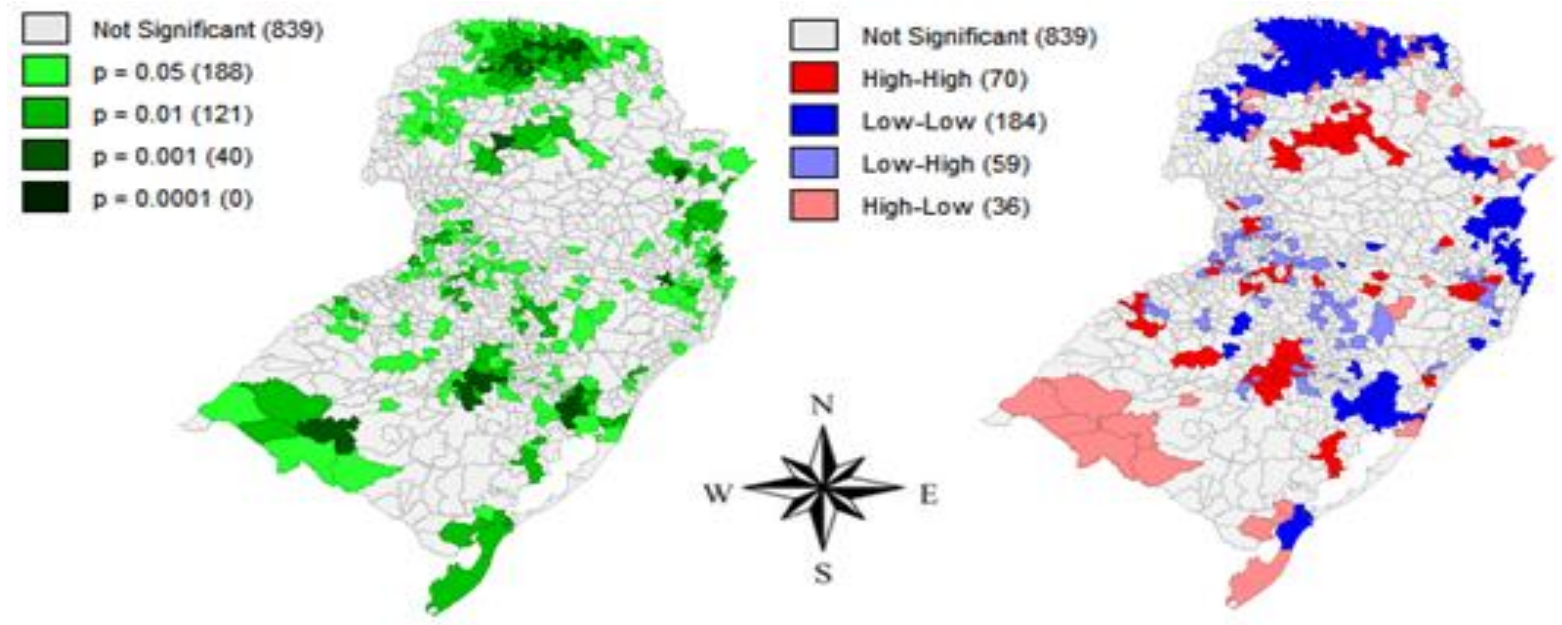

Fonte: Elaboração Própria com Dados do IBGE e Utilização do Software Geoda

Os mapas de cluster analisados anteriormente permitiram uma visualização geográfica dos agrupamentos espaciais significativos formados no território da região Sul do Brasil a partir da relação entre taxa de pobreza e percentual de população rural.

Em ambos os períodos analisados, ano de 2000 e de 2010 as aglomerações espaciais que exibem elevada pobreza e população rural aparecem concentradas na região Central do Paraná e no Rio Grande do Sul, enquanto que a situação oposta, ou seja, os regimes espaciais com baixa pobreza e população rural estão concentrados nas regiões do Norte do Paraná e Metropolitana de Porto Alegre, no Litoral do paranaense e em algumas localidades de Santa Catarina.

\section{CONSIDERAÇÕES FINAIS}

O presente artigo objetivou verificar se existência de relação espacial entre a taxa de pobreza e o percentual de população rural nos municípios da região Sul do Brasil no ano de 2000 e de 2010. Os resultados mostraram que a pobreza não se distribui de forma homogênea entre as unidades municipais da região Sul, assim, foram observadas regiões que contemplam taxa de pobreza acima da média.

Com a estatística I de Moran e com o diagrama de dispersão de Moran foi possível observar que a pobreza apresenta relação espacial positiva, assim, os municípios que exibem elevada taxa de pobreza tendem a estar rodeados por vizinhos na mesma situação e os municípios com taxa de pobreza abaixo da média tendem a estar circunvizinhados por municípios com situação semelhante. 
A analise bivariada permitiu verificar a relação entre a taxa de pobreza e a taxa de população rural dos municípios da região Sul do Brasil nos anos de 2000 e de 2010. Os resultados apontaram para relação espacial positiva, sendo assim pode se inferir que as unidades municipais que exibem elevada taxa de pobreza são circunvizinhadas por municípios com percentual de população rural acima da média.

Pode-se verificar também através da (AEDE), que entre os anos de 2000 e de 2010 houve aumento pouco expressivo da autocorrelação positiva entre o percentual da população rural e taxa de pobreza para os municípios da região Sul.

Os resultados da AEDE indicaram que os municípios com maiores percentuais de população rural e taxa de pobreza acima da média estão concentrados nas regiões Central do Paraná e do Rio Grande do Sul, já a concentração de municípios com os menores percentuais de pobres e de população rural estão localizados nas regiões do Norte do Paraná e Metropolitana de Porto Alegre nos dois anos analisados e no Litoral do Paraná e de Santa Catarina no ano 2000 e no Litoral de Santa Catarina no ano de 2010.

Foi observado aumento no número de municípios do tipo baixo-baixo no período, ao passo que foi verificado uma pequena redução do número de municípios do tipo alto-alto e do tipo alto-baixo, já a outra região atípica (baixo-alto) não houve mudanças no número de municípios.

Portanto, conclui-se que, mesmo havendo uma redução significativa da taxa de pobreza e da população rural no período de 2000 a 2010 na região Sul do Brasil, os municípios que concentram grande parte de sua população residindo em área rural continuam com taxa de pobreza acima da média reforçando os indícios de que a população rural tem dificuldade em usufruir de políticas de combate à pobreza.

\section{REFERÊNCIAS}

ALMEIDA, Eduardo. Econometria Espacial Aplicada. Editora alínea. 2012.

ANSELIN, L. Local indicators os spatial association - LISA. Geographical analysis, v. 27, n. 2, p. 93-115, 1995.

ANSELIN, L.; SYABRI, I.SMIRNOV, O. Visualizing multivariate spatial correlation with dynamically linked windows. University Illinois, 2003 (mimeo). 
CANCIAN, V.; VIDIGAL, V.G.; VIDIGAL, C.B.R. Pobreza e desigualdade de renda nos municípios da região sul do Brasil: uma análise espacial. In: XVI Encontro de Economia da Região Sul (ANPEC SUL), Curitiba, 2013.

DEDECCA, C.S. Contribuições para a agenda da política de combate à pobreza rural. In: Buainain, A. M.; DEDECCA, C.S. A nova cara da pobreza rural: desenvolvimento e a questão regional. Brasília: IICA, p. 19-32, 2013.

DINIZ, Sarah Silveira. Analise espacial da produtividade da Laranja dos municípios do estado de São Paulo: 2002 a 2010. Dissertação de Mestrado. Universidade Estadual de Londrina, 2012, 118 p.

HADDAD, E.A.; PIMENTEL, E.A. Análise da distribuição espacial da renda no estado de Minas Gerais: Uma abordagem setorial. Núcleo de Economia Regional e Urbana do Estado de São Paulo. Fevereiro de 2004.

HELFAND, S.M.; PEREIRA, V.F. Determinantes da pobreza rural e implicações para a política pública no Brasil. In: Buainain, A. M. A nova cara da pobreza rural: desafios para as políticas públicas. Brasília: IICA, p. 154-160, 2012.

IBGE - INSTITUTO BRASILEIRO DE GEOGRAFIA E ESTATÍSTICA. Disponível em http: //www.ibge.com.br, Instituto Brasileiro de Geografia e Estatística (IBGE). Acessado em 25 de novembro de 2014.

IPEA - INSTITUTO DE PESQUISAS ECONÔMICAS. Disponível em http://www.ipea.gov.br, Instituto de Pesquisa Econômica Aplicada (IPEA). Acesso em 26 de novembro de 2014.

IPEADATA. Instituto de Pesquisas Econômica Aplicada. Base de dados sociais. Disponível em:

<http://www.ipeadata.gov.br/> Acesso em: jun. 2012.

KAGEYAMA, A. As múltiplas fontes de renda das famílias agrícolas brasileiras. Agricultura em São Paulo, São Paulo v. 48, n. 2, p. 57-69, 2001.

MEDEIROS, E. R. ; BARBOSA, O. C. ; CAMARA, M. R. G. ; NASCIMENTO, S. P.; DINIZ, S. S. . Evolução do PRONAF e distribuição espacial da produtividade do milho e feijão na agricultura familiar no estado do Paraná para os anos de 2000 e 2010. In: $51^{\circ}$ Congresso da Sober, 2013, Belém. 51 Congresso da Sober, 2013.

MOCELIN, Cassia Engres. O Programa Bolsa Família enquanto principal estratégia de enfrentamento à pobreza rural no contexto brasileiro atual. In: XV Seminário Interinstitucional de Ensino, Pesquisa e Extensão da Unicruz. Universidade de Cruz Alta, novembro de 2010. Disponível em: 〈http://www.unicruz.edu.br〉. Acesso em 05 de Setembro de 2014.

NEDER, H.D.; SILVA, J.L.M. Pobreza e distribuição de renda em áreas rurais. Uma abordagem de inferência. RER, Rio de Janeiro, vol. 42, nº 03, p. 469-486, jul/set 2004.

ROCHA, Sonia. Pobreza no Brasil: afinal do que se trata? Rio de Janeiro, Ed. FGV, 2006. 
PAULA, J.S; DALBERTO, C.R; BOHN, L. Determinantes da pobreza rural no Brasil: um enfoque espacial. $6^{a}$ Conferência Internacional sobre Estatísticas Agropecuárias. Rio de Janeiro, 2013.

PEROBELLI, F.S.; ALMEIDA, E.S.; ALVIM, M.I.S.A.; FERREIRA, P.G.S. Análise espacial da produtividade do setor agrícola brasileiro: 1991-2003. Revista Nova Economia. Vol.17 no. 1, Belo Horizonte, p. 65-91, Jan./Apr. 2007.

ROCHA, S. Pobreza no Brasil: afinal do que se trata? Rio de Janeiro: Editora FGV, 2006.

ROCHA. S. Pobreza e renda - medidas per capita versus adulto equivalente. Rio de Janeiro: IBGE ECLAC, 1998. Disponível em: 〈http://www1.ibge.gov.br/poverty/pdf/s34s/rocha.pdf> Acesso em: 14/08/2014.

SANTOS, Larissa, Martins. Pobreza como privação de liberdade: Um estudo de caso na favela do Vidigal no Rio de Janeiro. (Dissertação de Mestrado) Rio de Janeiro, Universidade Federal Fluminense. Pós-Graduação em 2007. http://www.cpgeconomia.uff.br//novosite/arquivos/tese/2007-larissa_martins.pdf

SCHNEIDER, S.; FIALHO, M. A. V. Pobreza rural, desequilíbrios regionais e desenvolvimento agrário no Rio Grande do Sul. Teoria e Evidência Econômica, Passo Fundo, 2000 .

SEN, A. Desigualdade Reexaminada. São Paulo / Rio de Janeiro, Record, 2001.

SILVA, José Graziano. A nova dinâmica da agricultura brasileira. 2 ed. rev. Campinas: Unicamp, 1998. $211 \mathrm{p}$.

SILVA, M.de C.P. da; BARROS, R.P. de Pobreza multidimensional no Brasil. Anais da ANPEC Nacional 2006. Disponível em http.:// http://www.anpec.org.br/ encontro2006/artigos/A06A141.pdf. Acesso em 13 de outubro de 2014.

TEIXEIRA, K.H. Uma análise da distribuição espacial da pobreza nos municípios alagoanos. 2nd Ibero American Meeting on regional development and XII ENABER, Belo Horizonte, 2014. 